\title{
How to quantitate valve regurgitation by echo Doppler techniques
}

Iain A Simpson, Mark A de Belder, Antoinette Kenny, Michael Martin, Petros Nihoyannopoulos, on behalf of the British Society of Echocardiography

Echo Doppler techniques have revolutionised the non-invasive investigation of valvular heart disease and have become widely accepted and used as part of modern clinical cardiology. There is no single method that provides an entirely accurate quantitative assessment of the severity of valve regurgitation, and the complex interaction of anatomic and haemodynamic variables can add to these potential difficulties. Although cardiac ultrasound techniques do provide important clinical information in patients with valve regurgitation it is very important that the results are interpreted with a knowledge not only of the clinical findings but also of the advantages and limitations of cardiac ultrasound. This article attempts to provide a practical perspective of the current value of echo Doppler techniques in the assessment of valve regurgitation and to provide an insight into the relative merits of individual methods of quantitation.

\section{Basic principles of assessing valve regurgitation by echo Doppler techniques}

Absolute quantitation of regurgitation requires an accurate estimate of the regurgitant blood volume but this is not possible with current echo Doppler technology. However, even if we could easily estimate the true volume of regurgitation using current ultrasound technology, this may not necessarily provide the most relevant clinical information. The same volume of regurgitation may produce different haemodynamic effects in different patients. It is well recognised that symptoms are not directly related to the severity of regurgitation. In many cases it is the symptomatic result of regurgitation as well as the haemodynamic consequences in terms of ventricular function and the development of pulmonary hypertension that determine the need for surgical intervention. Quantitative assessment of valve regurgitation is therefore a far more complex issue than simply attempting to estimate the absolute volume of regurgitation, and must take into account the presence of chamber dilatation and function, valve structure, and the haemodynamic characteristics of regurgitant flow.

\section{ECHOCARDIOGRAPHY}

Two dimensional and $M$ Mode echocardiography provide no direct information on valve regurgitation, yet inferences about the severity of regurgitation can often be made from its effect on chamber size and function. Additionally, the high resolution imaging of modern echocardiography, including transoesophageal imaging, provides valuable information on disordered valve morphology. Significant chronic valve regurgitation causes volume overload in the receiving chamber which will eventually result in chamber dilatation.

\section{SPECTRAL DOPPLER ULTRASOUND}

Spectral Doppler ultrasound can accurately estimate flow velocity within the heart and great vessels. Continuous wave Doppler can estimate even very high velocities which are often associated with valve regurgitation, whereas spectral pulsed wave Doppler can more accurately localise the velocity values in relation to surrounding structural information. In neither case can absolute volume flow be measured directly, though it is possible to determine qualitative and semiquantitative information on the severity of regurgitation and obtain an indirect estimate of the regurgitant flow by combining echocardiography and Doppler techniques.

\section{COLOUR DOPPLER FLOW MAPPING}

Colour Doppler flow mapping allows Doppler information to be displayed in two dimensions in real time and in relation to surrounding structural detail. It is based on pulsed Doppler technology and is therefore subject to the inherent limitations of pulsed Doppler, such as aliasing and angle dependence. In visualising valve regurgitation, it is easy to fall into the trap of assuming that one is looking at an "ultrasonic angiogram" and that the spatial distribution of the regurgitant jet as seen on colour flow mapping is equivalent to the volumetric regurgitation of contrast angiography. However, as with spectral Doppler ultrasound, colour Doppler flow mapping displays velocity rather than volume information, and any volumetric component to the spatial registration of colour is derived rather than direct information.

\section{COMBINING ECHO DOPPLER INFORMATION}

One of the strengths of echo Doppler evaluation of valve regurgitation is the ability to derive information from a variety of different types of imaging, as described above. Each piece of data is important to a greater or lesser extent, and allows a much more accurate overall assessment of the severity of regurgitation than would be possible using only a single 


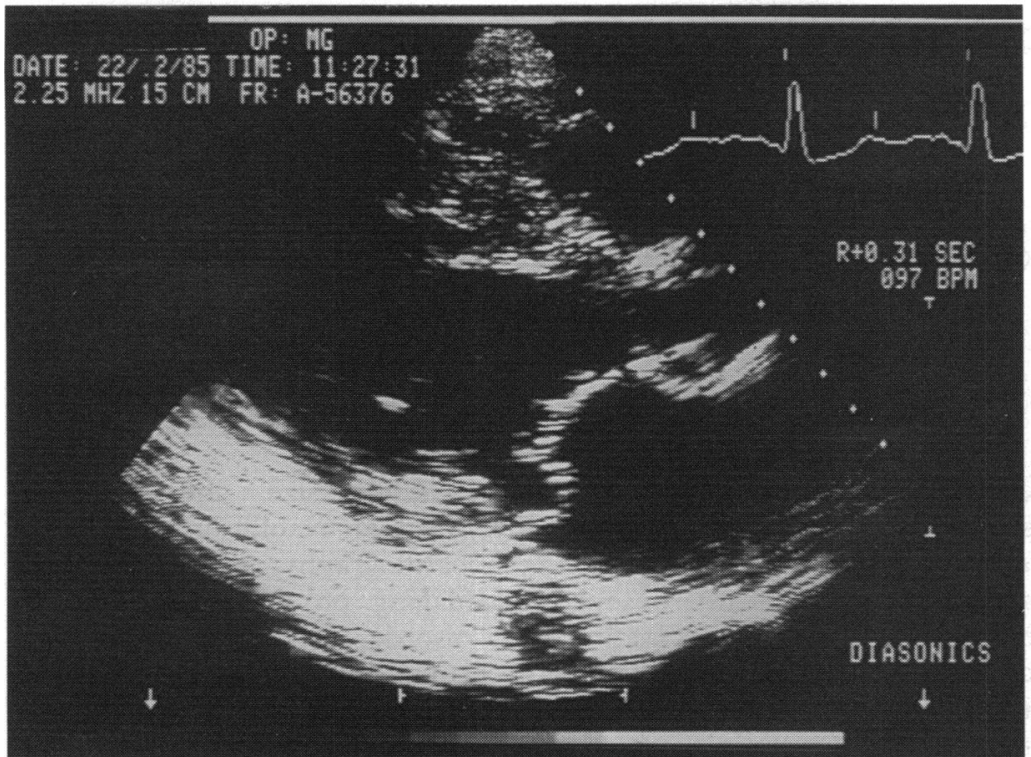

Figure 1 Two dimensional echocardiographic image from a patient with prolapse of the posterior mitral valve leaflet which can be seen protruding back into the left atrium on this parasternal long axis view.

aspect of echo Doppler technology. Similarly, it should be emphasised that the result of echo Doppler investigation is only one part of the clinical assessment of valve regurgitation and it is important to interpret the result in combination with the clinical history and examination, as well as other non-invasive and invasive investigations. The quality of the echo Doppler information is also an important issue. An echo Doppler study of high technical quality may provide precise and clinically useful information on the severity of regurgitation whereas overinterpretation of a suboptimal study may lead to errors in clinical judgement. In such cases more invasive investigations such as transoesophageal echocardiography or cardiac catheterisation may be required.

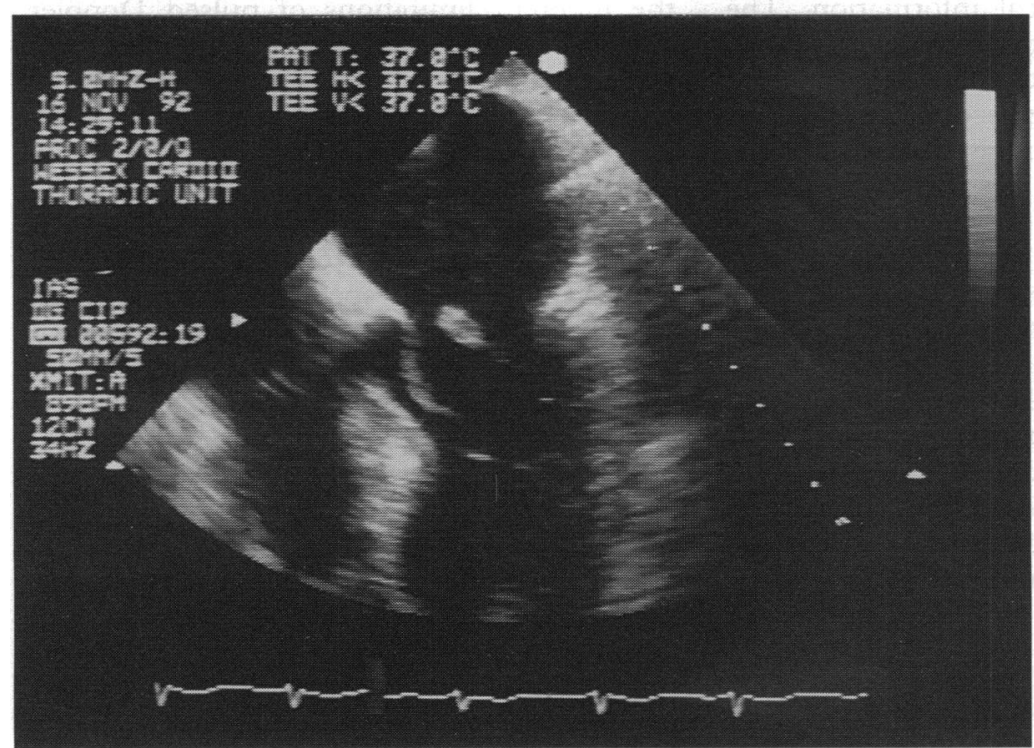

Figure 2 Papillary muscle rupture imaged by transoesophageal echocardiography. The head of the papillary muscle is imaged in the left atrium during systole with a resulting flail posterior mitral valve leaflet.

\section{Mitral regurgitation}

Extensive effort has been directed at the quantitation of mitral valve regurgitation by echo Doppler techniques as an adjunct to clinical assessment.

There is no single effective method of assessing mitral regurgitation in an individual patient using echo Doppler techniques. Although it is possible to indicate which aspects of cardiac ultrasound might provide the most useful and reliable methods, operator experience in discerning the image quality and the relative emphasis of individual results is still of paramount importance.

\section{ECHOCARDIOGRAPHY}

Quantitative information on the severity of mitral regurgitation using $M$ mode and two dimensional echocardiography is gained from the morphology of the mitral valve and from the haemodynamic effects of the mitral regurgitation on the left atrium and left ventricle.

\section{Mitral valve morphology}

One of the major strengths of echocardiography is its ability to outline the mitral valve with great accuracy. This is important in choosing the optimal surgical approach in reconstructive surgery where particular emphasis should be placed on obtaining a detailed description of the entire mitral apparatus by both transthoracic and transoesophageal echocardiography. ${ }^{1}$ The most common morphological abnormality associated with pure mitral regurgitation is myxomatous degeneration of the valve, often indicated by distinct prolapse of one or both of the valve leaflets (fig 1). The degree of prolapse and the amount redundant valve tissue do not necessarily correlate with the severity of regurgitation, but when this structural information is combined with the alterations in chamber dimensions and function indicated below, it is a strong indication of the presence of haemodynamically significant mitral regurgitation. Similarly, the presence of flail chordae tendineae on either transthoracic or transoesophageal echocardiography is almost always associated with severe mitral regurgitation. Rarely, partial or even complete rupture of a papillary muscle associated with an inferior (and often small) myocardial infarct can produce torrential acute mitral regurgitation. These patients are usually in profound pulmonary oedema, requiring mechanical ventilation. Under these circumstances transthoracic echocardiography is difficult, but transoesophageal echocardiography can provide a rapid accurate diagnosis from the structural information alone ${ }^{2}$ (fig 2).

\section{Alteration in chamber characteristics}

The presence of severe mitral regurgitation has significant haemodynamic effects, primarily on the left atrium and left ventricle. Left atrial dilatation is commonplace with mitral regurgitation, and a normal sized left atrium is not normally associated with significant regurgitation unless it is acute, in which case the valve appearance is likely to be grossly abnormal. This may not be fully appreciated without the use of transoesophageal imaging. The left ventricle is volume overloaded by the increased flow through the mitral valve (a combination of the normal left atrial inflow plus the regurgitant volume) and dilates progressively as a result. In severe mitral regurgitation, left ventricular function is dynamic with an increase in left ventricular ejection fraction and fractional shortening and this is a very useful indication that significant regurgitation exists, particularly in acute severe mitral regurgitation where the left atrium may be of normal size. If significant regurgitation remains uncorrected, left ventricular dysfunction can often develop over a period of years, when surgical correction carries an increased morbidity and mortality. 
Cautionary notes

The value of chamber dilatation and function is limited in the presence of coexisting mitral stenosis or in patients with left ventricular dysfunction from other causes such as myocardial infarction or dilated cardiomyopathy, both of which can be associated with mitral regurgitation. Here the emphasis on echocardiographic features is limited and spectral Doppler ultrasound combined with colour Doppler flow mapping is a more reliable indicator of regurgitation severity. In patients with acute severe mitral regurgitation there may be no increase in left atrial size.

\section{SPECTRAL DOPPLER ULTRASOUND}

Both pulsed and continuous wave Doppler have a significant role to play in quantitating mitral regurgitation, although the role of spectral pulsed Doppler has largely been superseded by the introduction of colour Doppler flow mapping. Historically, pulsed wave Doppler has been used to map the spatial distribution of the regurgitant jet within the left atrium, ${ }^{34}$ though this is now more easily achieved using colour Doppler flow mapping. The high velocity pansystolic jet imaged by continuous wave Doppler in a direction away from the transducer is quite characteristic of the presence of mitral regurgitation. The extreme sensitivity of continuous wave Doppler allows the identification of even the most trivial mitral regurgitation, often not apparent clinically, yet makes accurate quantitation more difficult. Severity of mitral regurgitation can be estimated by spectral pulsed and continuous wave Doppler ultrasound in several ways, for example by the intensity of the spectral signal, the shape of the spectral envelope, an increase in mitral inflow velocity, estimation of regurgitant fraction, the pulmonary venous flow velocity pattern, or estimation of pulmonary hypertension from velocity of tricuspid regurgitation.

\section{Spectral signal intensity}

The spectral Doppler signal intensity is probably the most useful of these but it is still only semiquantitative at best. In severe mitral regurgitation more blood leaks back through the mitral valve and hence more blood cells are available as ultrasound reflectors. The more the transmitted ultrasound signal is reflected back to the transducer the more intense the Doppler signal becomes. Hence, the intensity of the Doppler signal is to some extent an indication of the severity of regurgitation. Since the overall intensity of the Doppler signal is dependent on many factors including body habitus, ultrasound penetration characteristics, Doppler gain, and so on. It is more valuable to interpret the signal intensity as a function of some internal marker, such as forward mitral flow. ${ }^{5}$ If the signal of mitral regurgitation is of similar intensity to that of mitral inflow, this is a good indication of the presence of severe mitral regurgitation. Similarly, a faint regurgitant signal in the presence of intense mitral inflow is usually associated with trivial or mild mitral regurgitation. With experience, the use of the signal intensity can be a valuable adjunct to the clinical and other non-invasive methods of assessing mitral regurgitation severity.

Mitral inflow velocity

An increase in the mitral inflow velocity is quite a sensitive marker of severe mitral regurgitation in the absence of mitral stenosis and is also valid for mitral bioprosthetic regurgitation. If the mitral regurgitation is of haemodynamic significance, the volume of regurgitation combined with the normal mitral inflow will be sufficient to increase the peak diastolic velocity through the mitral valve. If the peak velocity is greater than $2 \mathrm{~m} / \mathrm{s}$, this is a highly sensitive indicator of the presence of severe mitral regurgitation, particularly in the presence of a bioprosthetic mitral valve replacement. ${ }^{6}$ This methodology is of course invalid in the presence of mitral valve stenosis, where the peak diastolic velocity is increased as a result of the obstruction across the mitral valve.

Shape of spectral envelope and regurgitant fraction

The use of both these techniques is possible but quantitative inaccuracies preclude their routine use in clinical practice. The principle of regurgitant fraction estimates for the mitral valve is as follows:-

Regurgitant fraction $=$

mitral orifice area $\times$ mitral VTI $)-($ LVOT area $\times$ LVOT VTI $)$ (mitral orifice area $\times$ mitral VTI)

$\times 100 \%$

where VTI $=$ velocity-time integral and LVOT $=$ left ventricular outflow tract. The left ventricular outflow tract diameter is usually measured from the parasternal views and its area calculated, assuming a circular outflow tract, as $\pi(\mathrm{D} / 2)^{2}$, where $\mathrm{D}=$ LVOT diameter.

If this technique is to be used clinically, it should only be undertaken in echo laboratories that have validated the methodology and are using it on a routine basis.

Pulmonary venous flow velocity pattern

Severe mitral regurgitation will cause a significant alteration in the flow velocity pattern imaged in the pulmonary veins. Whereas normal pulmonary venous flow would be directed towards the left atrium during systole, in severe mitral regurgitation there is systolic flow reversal, such that flow is directed away from the left atrium, a consequence of the regurgitant volume displacing flow backwards in the pulmonary veins. Since the pulmonary veins are located posteriorly within the mediastinum, they are distant from a transthoracic echo transducer, so it is rarely possible to determine pulmonary venous flow patterns accurately without the use of transoesophageal echocardiography. Where present, systolic flow reversal usually indicates severe regurgitation, particularly in a large left atrium, but its absence does not preclude the presence of severe regurgitation.

Estimation of pulmonary hypertension from velocity of tricuspid regurgitation

Although the estimation of pulmonary hypertension from the velocity of tricuspid regurgitation does not provide direct information on the severity of mitral regurgitation, it does give useful circumstantial evidence of its functional severity where mitral regurgitation is present as an isolated lesion. It is important to interpret carefully the velocity of tricuspid regurgitation since the presence of severe tricuspid regurgitation will reduce the velocity of regurgitation as the right atrial systolic pressure rises and it would then be possible to underestimate the true severity of pulmonary hypertension.

\section{COLOUR DOPPLER FLOW MAPPING}

The introduction of colour Doppler flow mapping was hailed as a method of providing an "ultrasonic angiogram" of mitral regurgitation, ${ }^{89}$ but its initial promise in this respect has not been fulfilled. Colour flow mapping is a complex display of velocity information related to surrounding structural detail, and although it has greatly enhanced our understanding of valve pathology and the flow dynamics of regurgitation it is not easy to extract information on the severity of regurgitation directly from the colour flow map image (fig 3).

What information can we obtain from 
Figure 3 Colour Doppler flow map image in the apical four chamber view. There is evidence of both mitral and tricuspid regurgitation with a central jet of mitral regurgitation and a broader jet of tricuspid regurgitation.

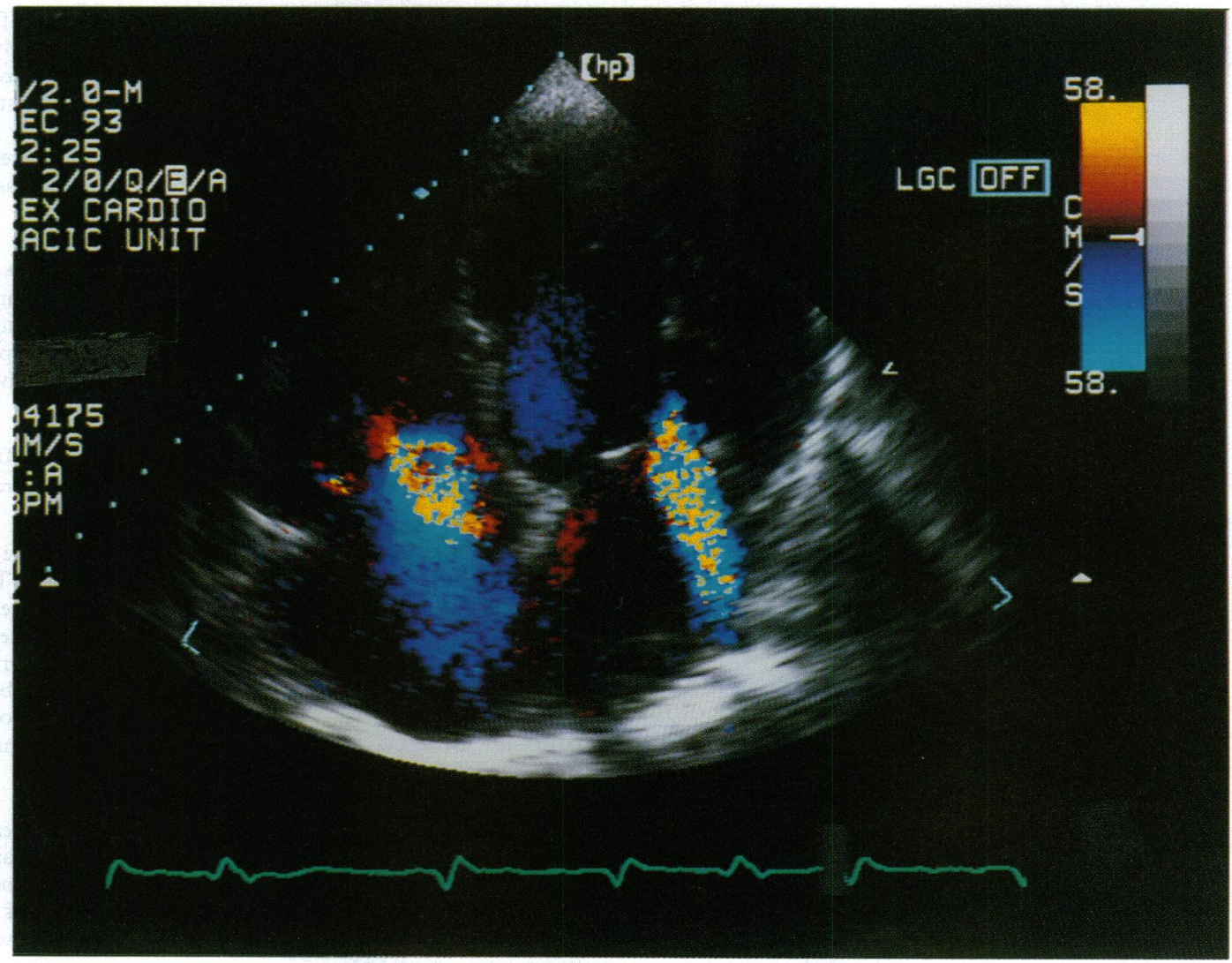

colour Doppler flow mapping of mitral regurgitation and how useful is this in quantitating its severity? It is important to remember that colour Doppler flow mapping is a velocity display and does not directly show volume information. It is also a pulsed Doppler technique and therefore subject to certain limitations including aliasing and angle dependence. Nevertheless, the spatial display of regurgitant jets and the relationship of this to valve morphology and chamber characteristics makes colour flow mapping an attractive method for estimating the severity of mitral regurgitation. There are several possible methods for quantitating mitral regurgitation using colour Doppler flow mapping. These are spatial jet distribution, basal jet characteristics, jet kinetic energy, jet momentum, and flow convergence estimates.

Spatial jet distribution

It would seem reasonable to assume that the size of the regurgitant jet within the left atrium would bear some direct relationship to the severity of regurgitation - the larger the jet the more severe the mitral regurgitation. Early reports of the use of colour flow mapping as an "ultrasonic angiogram" served to confirm that at least semiquantitation of mitral regurgitation was possible simply from measuring the maximum jet size within the left atrium. ${ }^{89}$ An improved evaluation can be achieved if the jet is imaged in two orthogonal planes and if the size of the jet in relation to the size of the left atrium is also taken into account. ${ }^{10}$ As a clearer understanding of the display characteristics of colour flow mapping became apparent, it is now generally accepted that there are many other factors that influence the dimensions of the regurgitant jet including the driving pressure of regurgitation, so that in patients with a high left ventricular systolic pressure, the jet of regurgitation on colour flow mapping will be considerably larger than in patients with a much lower left ventricular pressure. ${ }^{11}$ Other haemodynamic factors influence the size of the regurgitant jet, including the compliance of the left atrium, ${ }^{12}$ the left atrial systolic pressure, and particularly whether the jet is free within the left atrium or whether it is a "wall jet" interacting with the left atrial wall. ${ }^{13}$ Here the viscous energy losses result in a significantly smaller regurgitant jet for the same level of mitral regurgitation. In addition to the haemodynamic factors indicated above, the apparent size of the regurgitant jet can be influenced greatly by alteration of instrumentation factors, including colour flow gain, transducer frequency, pulse repetition frequency, pulse packet size, and the type of colour flow algorithm chosen for colour display. ${ }^{14}$

Colour Doppler flow mapping is so sensitive to even very small volumes of blood travelling at high velocity that it has been reported to show mitral regurgitation in as many as $30 \%$ of normal individuals with no clinical evidence of regurgitation. ${ }^{15}$ Here, the colour flow jet is usually very small $\left(<1 \mathrm{~cm}^{2}\right)$ but the separation between normal or "physiological" regurgitation and mild abnormal regurgitation is largely clinical. Despite the limitations of the technique, it is generally possible to separate mitral regurgitation into mild, moderate, or severe on the basis of the colour jet dimensions ${ }^{16}$ taking and the effect of wall jets versus free jets.

\section{Basal jet characteristics}

The difficulties encountered in using the overall jet dimensions have led to alternative methodologies. One of the simplest and most effective is to use colour flow mapping to look at the dimensions of the regurgitant jet near its origin close to the mitral valve. Since a larger regurgitant orifice will cause more severe mitral regurgitation, it is likely that the dimensions of the jet as it comes through the mitral valve will, to some extent at least, reflect the functional orifice size. Initial data would seem to indicate that this is indeed the case. The height of the jet, its width, or the jet area can be used and there is less dependence on the haemodynamic and instrumentation factors so important in the overall distribution of the jet within the left atrium. One of the difficulties with this technique is that the jet dimensions are small, even in severe regurgitation, and if the jet is not imaged exactly at the orifice or if image into account the influence of haemodynamic variables 
quality is suboptimal inaccuracies may be expected. This method of estimating the severity of mitral regurgitation is particularly useful when combined with transoesophageal imaging.

fet kinetic energy and jet momentum analysis

The difficulties of using jet dimensions have led to more sophisticated methods of analysing regurgitant jet characteristics in an attempt to extract the volume information that must be inherent in a colour flow map image. ${ }^{111718}$ Although these estimates have a theoretical attraction, again they are of limited clinical value.

Flow convergence estimates

It has been recognised only fairly recently that the acceleration of flow into the regurgitant orifice on the left ventricular side of the mitral valve can be visualised by colour Doppler flow mapping and that the information within this flow convergence zone can provide useful quantitative information. ${ }^{19}$ The attraction of using this velocity information on the left ventricular side of the mitral valve is considerable. Firstly, there is no problem of jet interaction with the wall of the left atrium. Also, accelerating flow is more stable and therefore laminar as opposed to the highly turbulent jet of mitral regurgitation within the left atrium. Since turbulence causes significant loss of energy any quantitation derived from velocity information within the regurgitant jet is likely to be subject to some error. The concept of using the zone of flow convergence as a method of quantitating mitral regurgitation is basically simple and assumes that all flow contributing to the zone of flow convergence will pass through the regurgitant orifice. Since the flow convergence zone is basically hemispherical with isovelocity hemispheres of diminishing size and increasing velocity as flow approaches the regurgitant orifice, it is possible to calculate flow rate as a function of velocity $(\mathrm{cm} / \mathrm{s})$ and surface area $\left(\mathrm{cm}^{2}\right)$, and this can be applied to the flow convergence region. The point of aliasing is taken as the most reliable measurement position, usually at the second alias, a point at which experimental evidence exists that the flow convergence zone is truly hemispherical. The area of the hemisphere can be calculated from its radius ( $r$ ) as the distance from the mitral valve to the position of aliasing using the equation $2 \pi \mathrm{r}^{2}$. The velocity is apparent from the colour flow map image as the known Nyquist aliasing velocity value, allowing easy calculation of regurgitant flow rate. As yet the use of the flow convergence zone as a method of providing accurate quantitation of mitral regurgitation remains experimental ${ }^{20}$ but if the initial promise $^{2122}$ is confirmed and an on line estimation of flow rate can be provided throughout systole, the potential exists for real time, accurate, and clinically valuable estimates of the severity of mitral regurgitation from colour Doppler flow map images.

\section{Acute mitral regurgitation}

The development of acute severe mitral regurgitation is usually the result of ischaemia, infection, chordal rupture in myxomatous degeneration, or trauma. There is little time for chamber enlargement because of the acute onset, so the major feature on two dimensional images that raises the level of suspicion is the presence of hyperdynamic left ventricular function even in the setting of a normal sized left ventricle and atrium. A flail or partially flail mitral leaflet may be visualised but often this is only apparent with transoesophageal imaging. Spectral Doppler ultrasound shows an intense signal of mitral regurgitation but with a peak velocity early in systole and a rapid fall in velocity during systole resulting from the severe regurgitation into a small non- compliant left atrium. Colour Doppler flow mapping is often unimpressive and can lead to a false sense of security. If the regurgitation is severe, and through a large regurgitant orifice, the jet of regurgitation is broad but not particularly turbulent, and this more laminar low velocity flow may be overlooked as resulting from the normal swirling systolic flow often seen within the left atrium. Once again, a high level of clinical suspicion may direct the echocardiographer to the somewhat subtle abnormalities associated with this important pathological condition.

\section{Aortic regurgitation}

The assessment of aortic regurgitation by echo Doppler techniques is best achieved by a combination of echocardiography, spectral Doppler, and colour Doppler flow mapping in a similar manner to that for mitral regurgitation, and many of the same principles apply. It should be recognised that although it is important to assess the severity of regurgitation, it is its effect on the left ventricle that determines the onset of symptoms and the timing of valve surgery. It is not uncommon for some patients to remain asymptomatic for many years with good left ventricular function and no significant left ventricular dilatation. However, once left ventricular dilatation and in particular left ventricular dysfunction have occurred, the ideal time for operative intervention has already been missed. The timing of aortic valve surgery in patients with aortic regurgitation is therefore based on many factors and here more than in other valve lesions is the serial assessment by echo Doppler techniques of particular value.

\section{ECHOCARDIOGRAPHY}

Aortic regurgitation is not imaged directly by conventional echocardiography, but its presence can be inferred from the presence of high frequency fluttering of the anterior mitral valve leaflet or the interventricular septum using $\mathrm{M}$ mode imaging. Prolapse of the aortic valve can occur, though this is a much less frequent occurrence in comparison to its mitral counterpart. Aortic valve prolapse can affect any of the three cusps of the valve and the prolapse is often easily identified using two dimensional imaging, which can also determine the particular cusp involved. As with the mitral valve, the apparent severity of the prolapse on two dimensional imaging does not necessarily reflect the severity of regurgitation.

\section{Alteration in chamber characteristics}

It is the effect of aortic regurgitation on the left ventricle that is particularly important in assessing the functional significance of regurgitation. Aortic regurgitation will cause a volume overload in the left ventricle resulting in left ventricular dilatation. Much has been made of this dilatation in determining the timing of aortic valve surgery. It has been suggested that an end systolic diameter of $5.5 \mathrm{~cm}$ or greater indicates the need for surgery ${ }^{23}$ but this is of questionable clinical value. The serial change in dimension is probably of more value as it is likely to be progressive, so that increasing left ventricular dimensions over a period of months or even years indicates the need to consider 


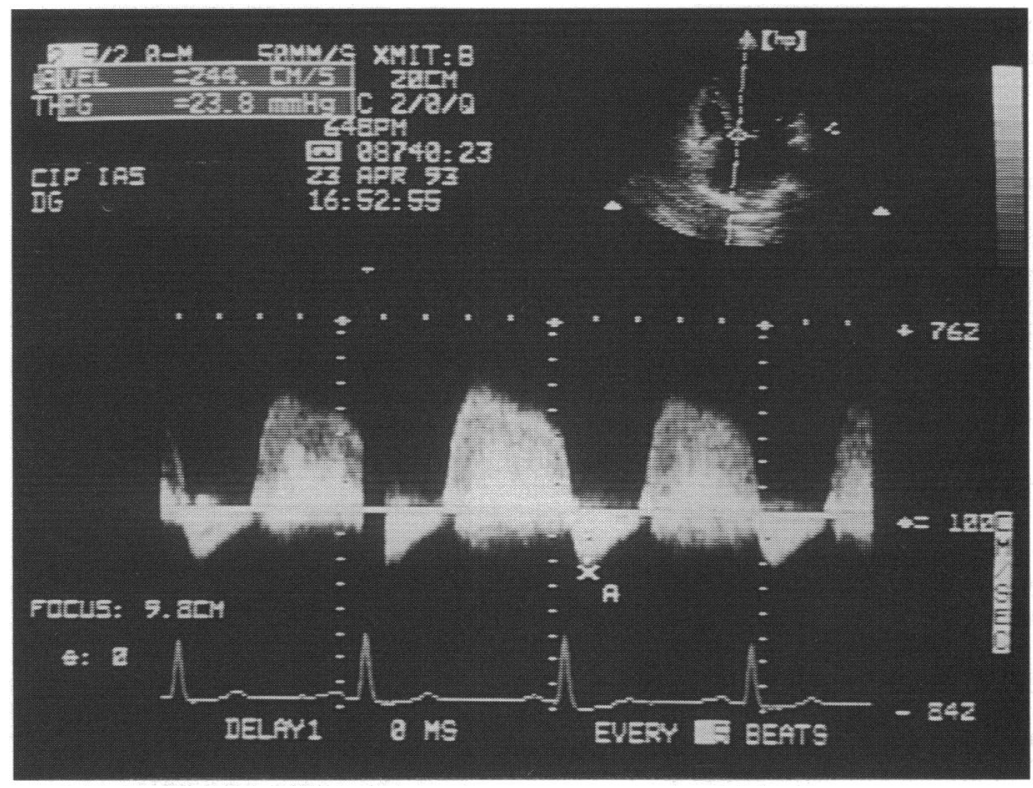

Figure 4 Spectral Doppler display of aortic regurgitation. There is an intense signal of aortic regurgitation throughout diastole coming towards the apically positioned transducer, as indicated by the velocity signal above the zero velocity line. In addition there is a rapid decrease in velocity during diastole indicating an equalisation of pressures. Both these features suggest the presence of severe regurgitation.

more definitive surgical intervention. One must interpret the dimensions, or indeed the changes in dimension, in context of other available clinical information and echocardiographic information, not least the presence and extent of left ventricular dysfunction. A decrease in left ventricular ejection fraction or fractional shortening is a far more worrisome feature and heralds the need for early surgery but is not always directly related to the severity of regurgitation. For example, the presence of concomitant coronary artery disease or previous myocardial infarction may cause a degree of underlying left ventricular dysfunction. In this context, even mild or moderate aortic regurgitation may be of greater clinical significance and precipitate symptoms and hasten further ventricular dysfunction. Early intervention may well be indicated even if the degree of aortic regurgitation is less severe than might otherwise merit surgery if aortic regurgitation were the sole pathology.

SPECTRAL DOPPLER ULTRASOUND

Spectral Doppler ultrasound, particularly continuous wave Doppler, is highly sensitive in detecting aortic regurgitation, and in elderly patients, often with somewhat thickened or even calcified aortic valves, aortic regurgitation is invariably present on continuous wave Doppler, though this is rarely apparent clinically. This extreme sensitivity does highlight the difficulty in using spectral Doppler ultrasound as a method of quantitating the severity of regurgitation. As with mitral regurgitation, the intensity of the spectral Doppler signal does provide a semiquantitative assessment of the severity of regurgitation, particularly when this is analysed in conjunction with the signal intensity of aortic systolic flow.

In severe aortic regurgitation there is a precipitant fall in the aortic diastolic pressure with an associated rapid rise in left ventricular diastolic pressure causing the maximum velocity of regurgitation to be rapidly reduced, often dramatically so (fig 4). The slope of the velocity curve is a reflection of this pressure equilibration and it is therefore possible to measure an aortic regurgitant half time in a similar way as for mitral stenosis, and use this as a quantitative assessment of the severity of regurgitation. However, in this case the shorter the pressure half time the more severe the regurgitation, such that a pressure half time of less than $300 \mathrm{~ms}$ is indicative of severe aortic regurgitation. ${ }^{24}$ Although a useful method of assessing regurgitation, and particularly providing an accurate numerical value for future comparison, there are several limitations to this technique. In particular, there may be quite a high pressure difference at end diastole in some patients with severe aortic regurgitation, so the presence of a long pressure half time does not exclude the presence of significant regurgitation, or pressure may rapidly equilibrate in the presence of left ventricular dysfunction erroneously suggesting more severe regurgitation. In severe aortic regurgitation, there is diastolic reversal of flow within the aorta and this can be detected using spectral Doppler. The presence of flow reversal in the descending aorta or even in the abdominal aorta is highly suggestive of severe regurgitation, but again the absence of flow reversal does not necessarily exclude the presence of significant regurgitation. It is also possible to estimate the regurgitant fraction in aortic regurgitation in a similar manner to that described above for mitral regurgitation, but again this is fraught with inaccuracies and is rarely applied in the clinical setting.

\section{COLOUR DOPPLER FLOW MAPPING}

Colour Doppler flow mapping is, like spectral Doppler, a highly sensitive clinically useful technique for detecting even trivial amounts of aortic regurgitation, but the question of quantitating its severity is far more complex. We have seen how difficult it is to use the spatial jet distribution as a marker of regurgitation severity for mitral valve regurgitation, and similar problems exist with aortic regurgitation. This is further compounded by the fact that the diastolic jet of aortic regurgitation will mix with left ventricular inflow through the mitral valve so the relative contribution of aortic regurgitation to the jet flow within the left ventricle will be lost. This makes the use of the spatial jet distribution on colour flow mapping of limited value, though a small narrow jet of regurgitation confined to the left ventricular outflow tract is almost always a reflection of trivial or mild regurgitation (fig 5). It is the width of the jet at its origin that is probably the most useful colour Doppler index of regurgitation severity. ${ }^{25}$ The possibility of using the flow convergence zone on the aortic side of the valve is still experimental but may further improve the accuracy of colour Doppler flow mapping estimates of aortic regurgitation.

\section{Maybe we should not be measuring anything?}

With the exception of chamber dimensions and left ventricular function assessed by 
Figure 5

Transoesophageal

echocardiogram in a

patient with mild aortic

regurgitation. The narrow

jet of regurgitation $(A R)$ is confined to the left

ventricular outflow tract. $L V=$ left ventricle; $A O=$ aorta; $L A=$ left atrium.

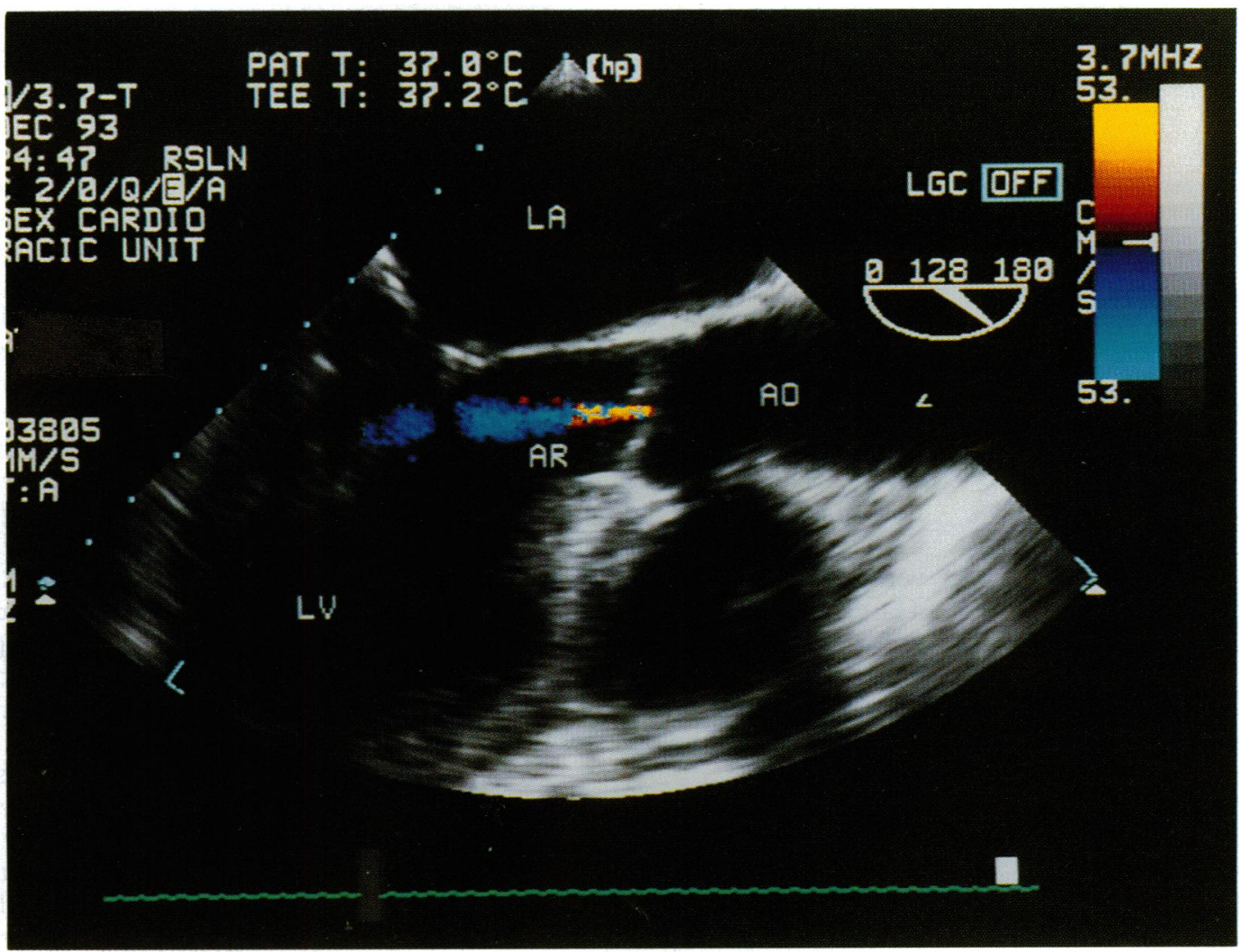

echocardiography all the methods of quantitating regurgitation are to some extent subjective and semiquantitative at best. Under these circumstances it may not be particularly useful to place numerical values on data that do not have the necessary accuracy or specificity, but rather to provide an overall assessment of the severity of regurgitation based on an appreciation of all the methods described above. In doing so a degree of subjectivity, interpretation, and operator experience is introduced, but since no single method provides the necessary quantitative information, this subjective evaluation is in practice inevitable. The different emphasis placed on the individual components of echocardiography, spectral Doppler, and colour Doppler flow mapping will depend on a number of factors, not least the quality of the echo study. If the patient is a poor echo subject then it is unlikely that quantitative information will be obtained from the study. Spectral Doppler ultrasound may be more helpful if a high quality continuous wave Doppler signal can be obtained even in the absence of satisfactory two dimensional echocardiographic imaging.

\section{Regurgitation of the right heart valves}

Modern cardiac ultrasound methods such as continuous wave spectral Doppler and colour Doppler flow mapping have changed our perspective of right heart valve regurgitation by showing that both the tricuspid and pulmonary valves are "physiologically" regurgitant in the vast majority of people with no discernible cardiac abnormality. ${ }^{26}$ Since the presence of regurgitation is an expected finding, quantitation of valve regurgitation is of some importance in an attempt to separate pathological from physiological regurgitation.

\section{Tricuspid regurgitation}

Chamber enlargement of both the right atrium and the right ventricle does occur with significant tricuspid regurgitation, but the presence of such chamber enlargement is generally the cause of rather than the effect of the tricuspid regurgitation. It is therefore rarely possible to use the chamber dimensions of the right heart to infer the severity of tricuspid regurgitation.

Spectral Doppler ultrasound can detect the presence of tricuspid regurgitation in the majority of individuals as indicated above. Using continuous wave Doppler it is often possible to estimate the peak velocity of regurgitation, which can be used to estimate the right ventricular systolic pressure ${ }^{27}$ but this is of little value in assessing the severity of tricuspid regurgitation. Although the systolic pressure difference between the right ventricle and atrium will increase in pulmonary hypertension, causing an increase in the peak velocity of tricuspid regurgitation, once severe regurgitation develops the right atrial pressure rises substantially and the pressure difference between the chambers falls, even though the absolute pressure remains high. The peak velocity of tricuspid regurgitation will fall as a result and may even return to "normal" values when the tricuspid regurgitation becomes severe. However, in this case the intensity of the Doppler signal will be high, reflecting the large regurgitant volume, and this combination of findings is a useful indicator of the presence of severe tricuspid regurgitation. 
In a similar fashion to the flow reversal seen in the pulmonary veins in severe mitral regurgitation, severe tricuspid regurgitation will cause systolic flow reversal in the superior and inferior venae cavae and even into the hepatic veins. This can easily be identified using either spectral Doppler ultrasound or colour Doppler flow mapping from the subcostal position.

Colour Doppler flow mapping can produce quite dramatic pictures in tricuspid regurgitation (fig 3) yet this is often more of a reflection of the presence of significant pulmonary hypertension than tricuspid regurgitation. In severe tricuspid regurgitation colour Doppler flow mapping can become quite unimpressive as there is free flow of blood from the right ventricle to the right atrium; although there is a large volume this is at a uniformly low velocity and therefore not particularly striking on colour flow mapping, being seen as a broad jet of low velocity "blue" flow away from the transducer from the parasternal or apical positions.

\section{Pulmonary regurgitation}

Two dimensional and $\mathbf{M}$ mode imaging have little to offer in terms of identifying the presence of pulmonary regurgitation or in providing quantitative information on its severity. Right ventricular or pulmonary artery dilatation, or both, may be present but these are rather non-specific features, more likely to be associated with pulmonary hypertension. Again, any such information is more likely to come from Doppler techniques, though similar problems exist to those discussed for tricuspid regurgitation.

Both spectral Doppler ultrasound and colour Doppler flow mapping are extremely sensitive in detecting the presence of pulmonary regurgitation and this is seen in over $90 \%$ of normal individuals if adequate ultrasound interrogation is used. ${ }^{26}$ Quantitation remains extremely difficult. The intensity of the Doppler signal will once again provide some semiquantitative assessment of severity, but the spatial jet distribution on colour Doppler flow mapping is directly related to the diastolic pressure difference between the pulmonary artery and the right ventricle. A large jet of regurgitation will normally be seen in the presence of pulmonary hypertension, but without a significant volume of regurgitation. As with severe tricuspid regurgitation, the Doppler features of severe pulmonary regurgitation may be rather unimpressive with free flow of regurgitation seen as a broad based jet of uniformly low velocity "red" flow towards the transducer from the parasternal position.

\section{Prosthetic valve regurgitation}

Prosthetic valve regurgitation merits some special mention, though much of the information on its assessment has already been published. ${ }^{28}$ Quantitation of bioprosthetic valve regurgitation is very similar to that of native valve regurgitation for both the mitral and aortic positions. Mechanical valves have by their nature a degree of inherent regurgitation, particularly at the hinge points which can be visualised by spectral Doppler and especially colour Doppler flow mapping. Attempting to quantitate the severity of mechanical valve regurgitation requires an understanding of the regurgitant "finger print" of each of the individual types of mechanical prosthesis. It is now generally recommended that patients with leaking mechanical valves be studied by transoesophageal echocardiography, particularly if the valve is in the mitral position.

Paravalvular regurgitation is always pathological and is due to valve dehiscence, often a result of infective endocarditis. The presence of paravalvular regurgitation on spectral Doppler or colour Doppler flow mapping is of considerably greater clinical significance that the actual severity of regurgitation which may be readily apparent from clinical or other ultrasound features, such as rocking of the valve prosthesis on two dimensional imaging. Paravalvular leaks are more likely to result in wall jets, that is, a jet of regurgitation which interacts with the wall of the receiving chamber producing smaller jets than might otherwise be expected from the haemodynamic severity of regurgitation. For this reason, one should always be cautious about suggesting that a paravalvular leak is of only mild or moderate severity.

\section{Conclusions}

Quantitation of valve regurgitation by echo Doppler techniques is a useful and well established part of routine clinical practice. It is highly dependent on the image quality in individual patients and it is important to recognise that no single technique provides precise quantitation in all patients or clinical circumstances. Operator experience and expertise in the interpretation of echo Doppler images is of paramount importance and the quantitative information provided by echocardiography, Doppler ultrasound, and colour Doppler flow mapping should be interpreted in the context of the clinical findings and other non-invasive information derived from individual patients.

1 Stewart WJ, Currie PJ, Salcedo EE, Klein AL, Marwick T, Agler DA, et al. Evaluation of mitral leaflet motion by echocardiography and jet direction by Doppler color flow mapping to determine the mechanisms of mitra regurgitation. F Am Coll Cardiol 1992;20:1353-61.

2 Himelman RB, Kusumoto F, Oten MK, Sh RB, Kusumoto F, Oken K, Lee E, Cahalan MK, Shah PM, et al. The flail mitral valve: echocardioimaging and Doppler color flow mapping. $\mathcal{f} \mathrm{Am} \mathrm{Coll}$ Cardiol 1991;17:272-9.

3 Quinones MA, Young JB, Waggoner AD, Ostojic MC Ribeiro LG, Miller RR. Assessment of pulsed Doppler echocardiography in detection and quantification of aortic and mitral regurgitation. Br Heart $¥ 1980 ; 44: 612-20$. 4 Blanchard D, Diebold B, Peronneau P, Foult JM, Nee M Guermonprez JL, et al. Non-invasive diagnosis of mitral regurgitation by Doppler echocardiography. Br Heart $\mathcal{J}$ 1981;45:589-93.

5 Utsunomiya T, Patel D, Doshi R, Quan M, Gardin JM. Can signal intensity of the continuous wave Doppler regurgitant jet estimate severity of mitral regurgitation? Am Heart f 1992;13:166-71. 
6 Simpson IA, Reece IJ, Houston AB, Hutton I, Wheatley DJ, Cobbe SM. Non-invasive haemodynamic assessment of 155 patients with bioprosthetic valves by ment of 155 patients with bioprosthetic valves by Doppler ultrasound: a comparison of the Wessex
Porcine, Low Profile Ionescu Shiley and Hancock Porcine, Low Profile Ionescu Shiley and Hanco

7 Rokey R, Sterling LL, Zohbi WA, Sartori MP, Limacher MC, Kuo LC, et al. Determination of regurgitant fraction in isolated mitral or aortic regurgitation by pulsed Doppler two-dimensional echocardiography. $f \mathrm{Am} \mathrm{Coll}$ Cardiol 1986;7:1273-8.

8 Omoto R, Yokote Y, Takamoto S, Kyo S, Ueda K, Asano $\mathrm{H}$, et al. The development of real-time two-dimensional acquired valvular diseases. With special reference to the evaluation of valvular regurgitation. Fpn Heart $\mathcal{F} 1984$; 25:325-40.

9 Miyatake K, Izumi S, Okamoto M, Kinoshita N, Asonuma $\mathrm{H}$, Nakagawa $\mathrm{H}$, et al. Semi-quantitative grading of severity of mitral regurgitation by real-time two-dimensional Doppler flow imaging techniques. $₹ \mathrm{Am}$ Coll Cardiol 1986;7:82-8.

10 Helmcke F, Nanda NC, Hsuing MC, Soto B, Adey CK, Goyal RG, et al. Color Doppler assessment of mitral regurgitation with orthogonal planes. Circulation 1987; 75:175-83.

11 Simpson IA, Valdes-Cruz LM, Sahn DJ, Murillo A, Tamura T, Chung KJ. Doppler color flow mapping of simulated in vitro regurgitant jets: evaluation of the
effects of orifice size and hemodynamic variables. $\mathcal{A m}$ Coll Cardiol 1989;13:1195-207.

12 Maciel BC, Moises VA, Shandas R, Simpson IA, Beltran $M$, Valdes-Cruz LM, et al. Effects of pressure and volume of the receiving chamber on the spatial distribution of regurgitant jets as imaged by color Doppler tion of regurgitant iets as imaged by color Doppler flow mas-13.

13 Chao K, Moises VA, Shandas R, Elkadi T, Sahn DJ, Weintraub R. Influence of the Coanda effect on color Doppler jet area and color encoding. In vitro studies using color Doppler flow mapping. Circulation 1992;85: 333-41.

14 Utsunomiya T, Ogawa T, King SW, Sunada E, Moore $\mathrm{GW}$, Henry WL, et al. Effect of machine parameters on variance display in Doppler color flow mapping. Am Heart f 1990;120:1395-402.

15 Yoshida K, Yoshikawa J, Shakudo M, Akasaka T, Jyo Y, Takao S, et al. Color Doppler evaluation of valvular regurgitation in normal subjects. Circulation 1988;78: $840-7$.

16 Spain MG, Smith MD, Grayburn PA, Harlamert EA, DeMaria AN. Quantitative assessment of mitral regurgitation by Doppler color flow imaging: angiographic and hemodynamic correlations. F Am Coll Cardiol 1989;13: 585-90.

17 Thomas JD, Liu CM, Flachskampf FA, O'Shea JP, Davidoff $R$, Weyman AE. Quantification of jet flow by momentum analysis. An in vitro color Doppler flow study. Circulation 1990;81:247-59.

18 Bolger AF, Eigler NL, Pfaff JM, Resser KJ, Maurer G. Computer analysis of Doppler color flow mapping images for quantitative assessment of in vitro jets. $\mathcal{F} \mathrm{Am}$ Coll Cardiol 1988;12:450-7.

19 Recusani F, Bargiggia G, Yoganathan AP, Raisaro A, Valdes-Cruz LM, Sung HW, et al. A new method for quantification of regurgitant flow rate using color flow Doppler imaging of the flow convergence region proximal to a discrete orifice: an in vitro study. Circulation 1991;83:594-604.

20 Shang J, Jones $M$, Shandas R, Valdes-Cruz LM, Murillo A, Yamada I, et al. Accuracy of flow convergence estimates of mitral regurgitant flow rates obtained by use of multiple color flow Doppler M-mode aliasing boundaries: an experimental animal study. Am Heart $\mathcal{f} 1993$; aries: an experi

21 Bargiggia GS, Tronconi L, Sahn DJ, Rucusani F, Raisaro A, De Servi S, et al. A new method for quantitation of mitral regurgitation based on color flow Doppler imaging of flow convergence proximal to regurgitant orifice. Circulation 1991;84:1481-9.

22 Vandervoort PM, Rivera JM, Dele D, Palacios IF, Dinsmore RE, Weyman AE, et al. Application of color Doppler flow mapping to calculate effective regurgitant orifice area. An in vitro study and initial clinical observations. Circulation 1993;88:1150-6.

23 Henry WL, Bonow RO, Borer JS, Ware JH, Kent KM, Redwood DR, et al. Observations on the optimum time for operative intervention for aortic regurgitation. Circulation 1980;61:471-9.

24 Samstad SO, Hegrenaes L, Skjaerpe T, Hatle L. Half time of the diastolic aortoventricular pressure difference by continuous wave Doppler ultrasound: a measure of the continuous wave Doppler ultrasound: a measure of the severity

25 Switzer DF, Yoganathan AP, Nanda NC, Woo YR, Ridgway AJ. Calibration of color Doppler flow mapping during extreme hemodynamic conditions in vitro: a foundation for a reliable quantitative grading system for aortic incompetence. Circulation 1987;75:837-46.

26 Maciel BC, Simpson IA, Valdes-Cruz LM, Recusani F, Hoit B, Dalton N, et al. Color flow Doppler mapping studies of "physiologic" pulmonary and tricuspid regurgitation: evidence for true regurgitation as opposed to a valve closing volume. $7 \mathrm{Am}$ Soc Echocardiogr 1991;4: 589-97.

27 Berger M, Haimowitz A, Van Tosh A, Berdoff RL, Goldberg $E$ Quantitative assessment of pulmonary hypertension hypension in patients with tricuspid regurgitation using continuous wave

28 Chambers J, Fraser A, Lawford P, Nihoyannopoulos P, Simpson I. Echocardiographic assessment of artificial heart valves: British Society of Echocardiography position paper. Br Heart $\mathcal{f}$ 1994;71(Suppl):6-14 